Revta brasil. Bot., São Paulo, V.23, n.3, p.283-290, set. 2000

\title{
Biologia floral e reprodutiva de Casearia grandiflora Camb. (Flacourtiaceae)
}

\author{
ADRIANA DE OLIVEIRA MACHADO ${ }^{1}$ e PAULO EUGÊNIO A.M. DE OLIVEIRA ${ }^{1,2}$
}

(recebido em 20 de outubro de 1999; aceito em 22 de março de 2000)

\begin{abstract}
Breeding biology of Casearia grandiflora Camb. (Flacourtiaceae)). The floral biology of Casearia grandiflora (Flacourtiaceae) was studied in a remnant of mesophyllous forest in the Parque do Sabiá, Uberlândia-MG. The species is an important understorey element in forests of this region, flowering during most of the year but more abundantly in March, April and May. Flowers are pale-green, $7 \mathrm{~mm}$ across, in sessile axillary heads. They are odourless, one-day flowers, with 10 free stamens adenate to the corolla base. Between the stamens there are receptacular appendages called inter-staminal disk lobes. The stamens and these structures form a cone around the pistil which retains the nectar. Anthesis occurs irregularly but mainly early in the morning. Nectar is relatively abundant $(4 \mu \mathrm{L})$ and with $38 \%$ of sucrose equivalents. Pollen is released during the warmest hours of the day, with high viability levels (96.6\%). Stigma is receptive at the same time. The most frequent visitor was the fly Ornidia obesa (Syrphidae), which was present on flowers most of the day, although other sporadic visitors such as Meliponinae bees, butterflies and other unidentified flies were also observed. Controlled hand pollinations showed the species is self-sterile and non-apomictic. However, pollen tubes were observed growing down to the ovary and penetrating the ovules in self-pollinated pistils, which suggest late-acting self sterility phenomena (post-fertilization) or inbreeding depression. Self-sterility in $C$. grandiflora contrasts with autogamous breeding systems observed in most studied myophillous forest species with similar generalist flower morphology occurring in central Brazil, but is similar to other neotropical woody species in the Flacourtiaceae.
\end{abstract}

RESUMO - (Biologia floral e reprodutiva de Casearia grandiflora Camb. (Flacourtiaceae)). A biologia de reprodução de Casearia grandiflora (Flacourtiaceae) foi estudada em um remanescente de mata mesófila do Parque do Sabiá, Uberlândia - MG. A espécie é importante no sub-bosque de matas da região, florescendo durante quase todo o ano, mas com maior abundância nos meses de março, abril e maio. As flores são branco-esverdeadas com cerca de $7 \mathrm{~mm}$ de diâmetro, dispostas em capítulos sésseis axilares. Não apresentam odor perceptível e duram apenas 1 dia. Possuem 10 estames férteis livres entre si e unidos à corola na base. Entre os estames existem estruturas pilosas originárias do receptáculo floral. Os estames e estas estruturas formam um cone em torno do pistilo, onde se acumula o néctar. A antese ocorre de forma irregular, principalmente no início da manhã. O néctar é relativamente abundante $(4 \mu \mathrm{L})$ e com concentração média de $38 \%$ de equivalentes de sacarose. O pólen é liberado nas horas mais quentes do dia, com alta viabilidade (96,6\%). Neste horário também ocorre a receptividade estigmática. O visitante mais freqüente foi a mosca Ornidia obesa (Syrphidae), que visita as flores durante quase todo o dia, embora tenham sido observados também outros visitantes casuais como abelhas Meliponinae, borboletas e outras moscas não identificadas. Polinizações controladas mostraram que a planta é auto-incompatível e não apomítica. No entanto, foram observados tubos polínicos crescendo até o ovário e penetrando os óvulos em flores autopolinizadas, sugerindo a ocorrência de fenômenos de auto-esterilidade de ação tardia (pós-fertilização) ou depressão de endogamia. A auto-esterilidade em $C$. grandiflora contrasta com a autogamia observada na maior parte das plantas miófilas e de flores generalistas ocorrendo nas florestas do Brasil central, mas é semelhante aos sistemas de reprodução de outras Flacourtiaceae lenhosas estudadas em florestas Neotropicais.

Key words - Flacourtiaceae, myophily, fly pollination, Syrphidae, breeding system

\section{Introdução}

As relações entre as flores e seus polinizadores são freqüentemente interpretadas como resultado de interações onde as estruturas florais estão adaptadas para otimizar o transporte de pólen e mediar a ação dos vetores (Faegri \& Van der Pijl 1979, Crepet 1983, Proctor et al. 1996). Estas interações estão associadas à grande diversidade de flores tropicais

1. Departamento de Biociências, Universidade Federal de Uberlândia, Caixa Postal 593, 38400-902 Uberlândia, MG, Brasil. Bolsistas CNPq.

2. Autor para correspondência: poliveira@ufu.br
(Bawa 1990, Endress 1994) e à biodiversidade tropical como um todo (Bawa 1974).

Sistemas de polinização envolvem desde insetos pouco especialistas até animais estritamente dependentes das flores para sua sobrevivência, como abelhas e mesmo vertebrados, como aves e morcegos. A polinização por moscas tem sido considerada irregular e incerta, pois esses insetos não alimentam sua prole com recursos florais, utilizando outras fontes alimentares, e porque as plantas visitadas por moscas são geralmente visitadas também por uma grande variedade de outros insetos (Proctor et al. 1996). No entanto, os dípteros podem ser importantes polinizadores, pois geralmente estão presentes ao longo de todo o ano, ao contrário de outros vetores, que podem apresentar períodos mais restritos de 
atividade (Faegri \& Van der Pijl 1979). Estes sistemas de polinização pouco especializados parecem ser mais comuns do que previamente se pensava (Bawa 1990) e a polinização por moscas tem sido freqüentemente observada em espécies importantes de florestas tropicais (Taroda \& Gibbs 1982, Pombal \& Morellato 1995).

Encontrada desde a Amazônia até o Estado de São Paulo, incluindo Minas Gerais, Goiás e Mato Grosso, Casearia grandiflora Camb. é uma árvore comum no sub-bosque de matas do Brasil central. Nesses ambientes, é uma espécie relativamente importante, aparecendo em diversos levantamentos fitossociológicos na região (Guilherme et al. 1998, Silva-Jr. et al. 1998).

Este trabalho teve como objetivo estudar a biologia floral, polinização e a reprodução de Casearia grandiflora a fim de melhor entender seu comportamento reprodutivo e compará-la com outras espécies de semelhante biologia reprodutiva.

\section{Material e métodos}

A área de estudo é um remanescente de mata mesófila do Parque do Sabiá, que se localiza no perímetro urbano do município de Uberlândia-MG $\left(18^{\circ} 55^{\prime} \mathrm{S}\right.$ e $48^{\circ} 17^{\prime} \mathrm{W}$, altitude aproximada de $890 \mathrm{~m}$ ). O clima na região pode ser caracterizado como Aw megatérmico, com estações de seca e chuva bem definidas, com precipitação anual em torno de $1550 \mathrm{~mm}$ e temperatura média de $22{ }^{\circ} \mathrm{C}$ (Rosa et al. 1991). Nesta mata, Casearia grandiflora tem distribuição regular, apresentando as maiores taxas de frequiência e densidade relativas e de IVI (Índice de Valor de Importância), quando comparada com as demais espécies presentes na área (Guilherme et al. 1998).

Nos meses de março a junho de 1996, flores de 14 plantas marcadas no campo foram acompanhadas para observar sua longevidade e demais eventos florais. Algumas observações foram completadas posteriormente. Um exemplar herborizado da espécie foi depositado no Herbarium Uberlandense (HUFU $\mathrm{n}^{\circ}$ 13.809).

Foi analisado o hábito das plantas floridas, quantidade de flores por inflorescência bem como o número das inflorescências por ramo. Foi observada, ainda, a disposição de flores na planta, horário de antese, período de disponibilidade de néctar e pólen, bem como de receptividade estigmática. Algumas flores foram fixadas em etanol 70\% para estudos morfológicos e para estimativas da viabilidade polínica. A análise da viabilidade polínica foi realizada com grãos de pólen retirados de anteras provenientes de flores de quatro indivíduos diferentes, corados com carmim acético (Kearns \& Inouye 1993). Algumas flores fixadas foram dissecadas para se fazer uma estimativa da proporção entre grãos de pólen e óvulos (Cruden 1977).

Microcapilares de vidro foram utilizados para coleta e avaliação do volume médio de néctar produzido em flores previa- mente ensacadas. Um refratômetro de mão foi utilizado para estimar sua concentração de equivalentes de sacarose (Inouye et al. 1980). Para localização dos tecidos nectaríferos das flores, estas foram submergidas em solução de vermelho neutro (1:10.000; Kearns \& Inouye 1993).

Espécimes dos visitantes florais foram coletados com redes entomológicas para identificação posterior e verificação da presença de pólen no corpo. Para tanto, utilizou-se gelatina glicerinada para retirar grãos de pólen do corpo dos animais coletados e coloração posterior com carmim acético (modificado de Radford et al. 1974, Kearns \& Inouye 1993). A descrição do comportamento dos insetos visitantes foi feita através de observações diretas e análise de fotografias. Foram feitas observações durante todo o período de estudo, mas estas observações não foram quantificadas. Foi possível, no entanto, anotar as plantas onde os diferentes visitantes foram observados.

Inflorescências de cinco indivíduos na área de estudo foram previamente isoladas, um ou dois dias antes da antese das flores, utilizando-se sacos de organza de náilon. Estas inflorescências foram utilizadas para os seguintes tratamentos: autopolinização (feita depositando pólen de uma flor sobre seu próprio estigma); polinização cruzada (colocando-se no estigma pólen proveniente de flores de outros indivíduos distantes pelo menos $50 \mathrm{~m}$ ) e emasculação para testar apomixia (feita retirando-se os estames de botões em pré-antese e mantendo as flores isoladas até o fim da receptividade estigmática). Também foram feitas marcações em flores para a observação da eficácia da polinização natural (controle). O sucesso dos tratamentos e da polinização natural foi estimado em termos do percentual de frutos iniciados (anotados no primeiro mês após a maioria das polinizações terem sido realizadas) e daqueles que chegaram à maturação (no final do mês de junho, quase 3 meses após as polinizações), em relação ao número de flores tratadas.

Após os tratamentos de polinização controlada, algumas flores foram fixadas em álcool 70\%, a diferentes intervalos de tempo ( 24 e $48 \mathrm{~h}$ ), para posterior análise de germinação dos grãos de pólen no estigma e formação de tubos polínicos. Os pistilos foram observados utilizando-se a técnica de microscopia de fluorescência (adaptada de Martin 1959).

\section{Resultados}

$\mathrm{Na}$ área de estudo, Casearia grandiflora é uma árvore de até $8 \mathrm{~m}$ de altura, cuja floração ocorreu tanto em indivíduos de pequeno porte (desde $1 \mathrm{~m}$ de altura) como em indivíduos maiores. A floração ocorreu com maior intensidade entre os meses de março e maio. As flores estão dispostas em capítulos axilares ao longo dos ramos $(\overline{\mathrm{X}}=8 \pm 1,0 ; \mathrm{n}=12)$, mais concentrados na parte terminal. Cada capítulo é formado por 3 a 17 flores sésseis $(\bar{X}=9 \pm 1,1$; $\mathrm{n}=12$ ).

As flores são hermafroditas, diclamídeas, com corola pentâmera e pétalas unidas na base. Apresentam simetria radial e são do tipo aberto. O cálice é 
formado por 5 sépalas livres. As flores são brancoesverdeadas, opacas e têm cerca de 7,0 mm de diâmetro (figura 1a, ver também figura $2 \mathrm{a}$ ).

$\mathrm{O}$ androceu é composto por 10 estames férteis, em dois verticilos, sendo um deles mais baixo que o outro. Os estames são livres entre si, unidos à corola na base e exertos. Entre eles há estruturas pilosas, de tamanho menor, denominadas por Barroso et al. (1978) afigurações inter-estaminais do disco, originárias do receptáculo floral, que são características do gênero Casearia. Estas afigurações formam, juntamente com os estames, uma espécie de cone em torno do pistilo e abaixo do estigma (figura 1b). Os pelos no ápice destas estruturas são maiores e voltados para o interior e para baixo. As anteras têm deiscência longitudinal e são dorsifixas. $O$ conectivo forma uma massa globosa provavelmente de natureza glandular, que, no entanto, não se corou em vermelho neutro.

O ovário é súpero e o estilete é terminal. De maneira semelhante aos estames e às estruturas existentes entre eles, o pistilo é totalmente piloso, com pelos recobrindo até mesmo o estigma. $\mathrm{O}$ gineceu é sincárpico, tricarpelar unilocular, com placentação parietal.

A antese ocorreu de forma irregular, mas principalmente no início da manhã. O odor das flores foi imperceptível ao observador. As flores permanecem abertas durante apenas 1 dia. Ao final da tarde, estando as flores fertilizadas ou não, as pétalas se fecham e a flor retoma aspecto semelhante ao do botão, sendo, no entanto, maiores e mais escuras que estes. As pétalas e as demais peças florais persistiram
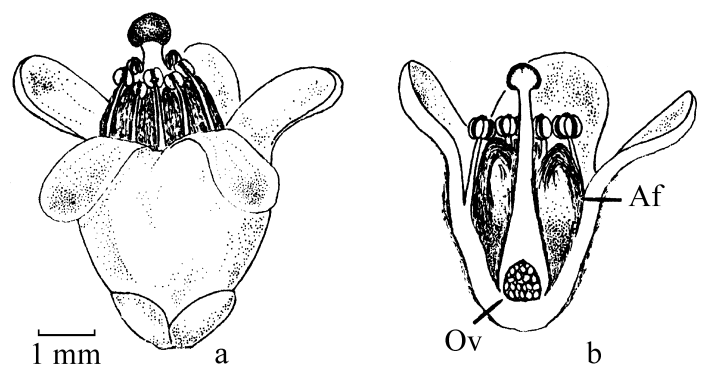

Figura 1. Morfologia floral de Casearia grandiflora. a) aspecto geral da flor aberta; b) corte longitudinal mostrando a estrutura da flor, com afigurações inter-estaminais (Af) e o ovário (Ov). secas até mesmo depois do início da formação do fruto, sem sofrer abscisão.

A receptividade estigmática tornou-se mais evidente nas horas mais quentes do dia, entre 11 e $15 \mathrm{~h}$, quando o estigma fica amarelado, brilhante e úmido (figura 2a). A disponibilidade de pólen também ocorreu neste horário. O pólen, amarelo e pegajoso, é liberado em grãos isolados e com viabilidade média de $96,6 \%( \pm 0,8 ; n=4)$. Cada flor apresentou em média 46 óvulos ( $\pm 2,7 ; n=5)$, e 3.694 grãos de pólen $(369,4 \pm 45,7$ grãos por antera; $n=5)$, o que confere uma relação pólen/ovulo de aproximadamente 82:1.

O néctar é relativamente abundante $(4 \mu \mathrm{L} \pm 0,4$; $\mathrm{n}=5)$ e com 38,8\% ( $\pm 3,9 ; \mathrm{n}=5)$ de concentração em equivalentes de sacarose, para medidas feitas no início da tarde. Uma medida realizada pela manhã apresentou concentração de apenas $15 \%$, indicando que a concentração de açúcares no néctar deve aumentar, provavelmente por efeito da evaporação, até o início da tarde. O néctar fica localizado no interior do cone formado pelos estames e pelas afigurações do disco. O teste feito com vermelho neutro não evidenciou as estruturas produtoras do néctar.

$\mathrm{O}$ visitante mais freqüente em Casearia grandiflora foi a mosca Ornidia obesa Fabricius, da familia Syrphidae. Estas moscas foram observadas repetidamente em todas as plantas acompanhadas durante o estudo e visitavam as flores durante quase todo o dia. Estas moscas caminhavam sobre as inflorescências até encontrarem flores abertas. Para retirar o néctar das flores, as moscas introduziam seu aparelho bucal entre o pistilo e os estames, de modo que o pólen podia ficar aderido a ele e à parte inferior do seu corpo (figura $2 \mathrm{~b}$ ). As moscas podiam passar vários minutos visitando um mesmo capítulo e podiam mover-se, sem voar, ao longo do ramo para outros capítulos. Quando não estavam sobre as flores ou pairando próximo a elas, esses insetos pousavam na face abaxial das folhas e permaneciam ali por longos intervalos de tempo. Nesse momento, muitas vezes limpavam seu aparelho bucal com o primeiro par de pernas. Raramente foram vistos movimentos das moscas entre plantas, mesmo plantas vizinhas. Foram também observados outros visitantes casuais, como abelhas Meliponinae (figura 2c), borboletas e outras moscas não identificadas. Estes visitantes fo- 

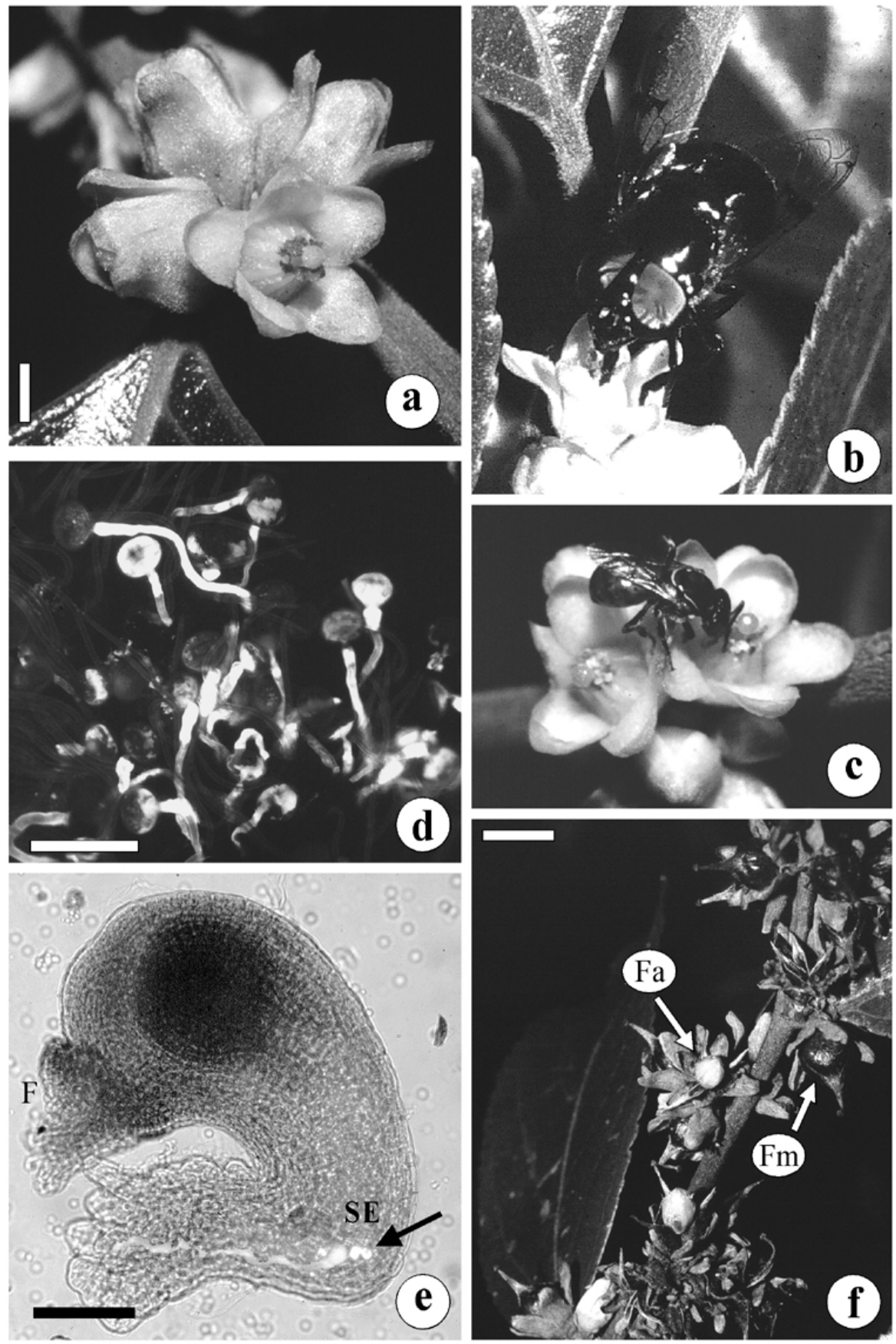

Figura 2. Biologia floral de Casearia grandiflora. a) Inflorescência com uma flor aberta, flores velhas já fechadas e botões. Escala $=2$ mm; b) Ornidia obesa (Syrphidae) visitando flores de C. grandiflora; c) Paratrigona cf. lineata Lepeletier (Apidae-Meliponinae) visitando flor de $C$. grandiflora; d) Parte do estigma de C. grandiflora mostrando grãos de pólen germinando entre os pelos que recobrem toda a superfície estigmática. Escala $=0,1 \mathrm{~mm}$; e) Óvulo de $C$. grandiflora em pistilo fixado $48 \mathrm{~h}$ após autopolinização. A seta indica a extremidade do tubo polínico penetrando o óvulo. Esta posição corresponde a extremidade do saco embrionário (SE) no óvulo maduro. F mostra a posição do funículo. Escala $=0,1 \mathrm{~mm}$; f) Frutos de $C$. grandiflora. Frutos maduros ainda fechados (Fm) e frutos abertos expondo o arilo alaranjado $(\mathrm{Fa})$. Escala $=1,0 \mathrm{~cm}$. 
Tabela 1. Resultados de frutificação dos diferentes testes de polinizações manuais realizados em Casearia grandiflora.

\begin{tabular}{lccc}
\hline Tratamentos & $\mathrm{n}^{\text {o }}$ de tratamentos & Frutos iniciados & Frutos maduros \\
\hline Autopolinização & 41 & $10(24 \%)$ & $0(0 \%)$ \\
Polinização cruzada & 45 & $22(49 \%)$ & $22(49 \%)$ \\
Emasculação & 38 & $\mathrm{nq}$ & $0(0 \%)$ \\
Controle & 156 & $50(32 \%)$ & $50(32 \%)$ \\
\hline
\end{tabular}

nq - não quantificado.

ram observados em apenas quatro plantas e raramente as observações se repetiram. A abelha Paratrigona cf. lineata, por exemplo, foi observada apenas numa planta e nunca voltou a ser observada durante o período de estudo.

A análise dos pistilos revelou que, após 24 h, os grãos de pólen germinavam profusamente em ambos os tratamentos (figura 2d) e os tubos polínicos já estavam chegando ao ovário nos testes de polinização cruzada $(n=4)$. Na autopolinização, os tubos parecem ter crescimento mais lento, estando ainda $3 / 4$ da distância até o ovário $(n=5)$. Após 48 h pode-se observar tubos polínicos penetrando óvulos em testes de autopolinização $(\mathrm{n}=4$; figura $2 \mathrm{e})$.

O início da formação de frutos por autopolinização ocorreu em $24 \%$ dos casos (tabela 1). Estes frutos se desenvolveram até certo ponto, mas não alcançaram a maturação. No primeiro censo realizado um mês após as polinizações, frutos de autopolinização e polinização cruzada apresentavam ovário expandido a ponto de afastar as pétalas remanescentes. Entretanto, no censo final, realizado dois meses após o primeiro, os poucos frutos remanescentes de autopolinização se apresentavam pequenos e murchos, caindo subsequentemente. Os frutos formados por polinização cruzada tiveram maior sucesso (49\%), com desenvolvimento integral até a maturação. Os frutos depois de maduros apresentavam coloração verde escura e se abriam expondo um arilo umedecido de cor laranja, bastante atrativo (figura 2f). Algumas flores emasculadas tiveram um início de desenvolvimento dos frutos (não quantificado quando do censo inicial), mas nenhum apresentou desenvolvimento completo. Nas flores marcadas como controle, houve uma considerável produção de frutos $(32 \%)$, que se desenvolveram até a maturação.

\section{Discussão}

As flores de Casearia grandiflora apresentam características como coloração clara e opaca, odor imperceptível, néctar de fácil acesso e órgãos sexuais expostos, que têm sido associadas à síndrome de miiofilia (Faegri \& Van der Pijl 1979). Estas características, entretanto, não limitam a polinização às moscas e as flores podem ser consideradas, até certo ponto, promíscuas, podendo atrair uma grande diversidade de visitantes oportunistas a procura de néctar e pólen facilmente acessíveis. A ocorrência de polinização por moscas em espécies arbóreas de flores aparentemente generalistas tem sido observada para outras árvores tropicais com flores pequenas (Pombal 1994, Pombal \& Morellato 1995) e sugere que esses insetos podem ser importantes para a polinização nesses ambientes.

As moscas de várias famílias são descritas como visitantes e polinizadoras de flores. Entretanto, Syrphidae é considerada a família de moscas visitantes de flores mais importante entre os Diptera (Proctor et al. 1996), se alimentando de pólen e néctar (Gilbert 1981, 1985, Owem 1981, Vockeroth \& Thompson 1987) e sendo, portanto, bons polinizadores potenciais.

Em estudo feito por Arruda \& Sazima (1996) para mata mesófila do estado de São Paulo, os Syrphidae utilizaram como recurso alimentar 58 espécies de plantas. Nas 31 espécies de plantas observadas mais detalhadamente, os Syrphidae foram os visitantes mais freqüentes em oito delas, competindo com outros dípteros e abelhas. Muitas destas flores possuem morfologia semelhante às de C. grandiflora. Espécies de Metrodorea têm flores do tipo aberto, com coloração clara e simetria radial (Pombal 1994). Entretanto, nestas flores o néctar fica exposto, enquanto que em $C$. grandiflora ele fica 
protegido pelo cone formado pelos estames e afigurações do disco. As flores de Dendropanax cuneatum também são pequenas, hermafroditas, esverdeadas e do tipo aberto exalando um odor levemente perfumado (Pombal \& Morellato 1995).

A facilidade de acesso ao pólen e néctar, e a coloração clara das flores, são responsáveis pela atração de sirfídeos e outras moscas às flores que não possuem odor marcado. O contrário ocorre com as moscas atraídas por flores sapromiiófilas, que geralmente são escuras e apresentam odor forte (Proctor et al. 1996). A maioria destas flores poderiam ser definidas como "inseto-generalistas" (Arruda \& Sazima 1996), sendo visitada por uma grande diversidade de insetos. Esta aparente promiscuidade e grande diversidade de insetos visitantes em outras plantas estudadas, contrasta com a dominância de Ornidia obesa em Casearia grandiflora. É possível que esta dominância seja um fenômeno restrito ao local de estudo, mas situação semelhante ocorre em Casearia sylvestris, uma espécie de cerrado que também é polinizada principalmente por moscas (Barbosa 1997).

Moscas e abelhas parecem ter preferência por flores com alta concentração de açúcares no néctar (Vogel 1983). Em C. grandiflora, a concentração de açúcares no néctar aumenta ao longo do dia, talvez devido à evaporação. O período de maior visitação coincide também com o período de maior concentração de açúcar durante a tarde, mas parece estar associado também à temperatura ambiente, diminuindo ao entardecer. Essa relação entre número de visitas e temperatura também foi verificada em Metrodorea (Pombal 1994) e em Dendropanax cuneatum (Pombal \& Morellato 1995).

Embora apenas ocasionalmente tenha-se observado diretamente o movimento das moscas entre plantas diferentes, a grande porcentagem de frutos formados no controle indica uma eficiência relativamente grande $(32,1 \%)$ na produção natural de frutos em C. grandiflora. Metrodorea nigra e $M$. stipularis, por exemplo, que são espécies também visitadas por moscas e produzem grande quantidade de flores, apresentam menor sucesso de frutificação (6 e 16\%, respectivamente; Pombal 1994).

A relação pólen/óvulo obtida para $C$. grandiflora sugere um sistema de reprodução entre autogamia obrigatória e facultativa (Cruden 1977). No entanto, diante dos resultados obtidos neste trabalho, pode-se considerar $C$. grandiflora como sendo obrigatoriamente xenogâmica, pois os frutos resultantes de autopolinização não se desenvolvem até a maturação. A presença de tubos polínicos e de óvulos penetrados nos testes de microscopia de fluorescência indica que não existe um sistema de autoincompatibilidade clássico que impeça a germinação dos grãos de pólen, crescimento de tubos polínicos, ou mesmo a penetração dos óvulos. O que parece ocorrer, é um fenômeno de auto-esterilidade (ou auto-incompatibilidade tardia) que impede o desenvolvimento e maturação dos frutos formados por autopolinização (Seavey \& Bawa 1986, Sage et al. 1994). Estes mecanismos são muito comuns em árvores tropicais (Seavey \& Bawa 1986, Oliveira \& Gibbs 2000) e mesmo em plantas miiófilas como Sterculia chicha (Taroda \& Gibbs 1982). O desenvolvimento irregular de frutos sugere que esta autoesterilidade possa estar associada à depressão endogâmica e não a mecanismos de incompatibilidade bem definidos (Wiens et al. 1987, Oliveira et al. 1991, Nic-Lughadha 1998, Gibbs et al. 1999).

A diferença na velocidade de crescimento dos tubos polínicos nos testes de autopolinização, em relação aos de polinização cruzada, não é tão marcada em Casearia grandiflora quanto em Dendropanax cuneatum. Nesta espécie autocompatível, os tubos polínicos de polinização cruzada chegam ao ovário depois de $36 \mathrm{~h}$, enquanto que em autopolinização somente após $60 \mathrm{~h}$. Esta diferença aumenta a probabilidade de formação de frutos por polinização cruzada (Pombal \& Morellato 1995).

Auto-esterilidade ou auto-incompatibilidade, com mecanismos clássicos ou não, é amplamente distribuída entre as espécies arbóreas tropicais, que são predominantemente xenógamas obrigatórias (Bawa 1974, Gibbs \& Bianchi 1999, Oliveira \& Gibbs 2000). Outras árvores neotropicais de Flacourtiaceae, como duas espécies de Casearia na Costa Rica (Kress \& Beach 1994) e Prockia flava na Venezuela (Ruiz \& Arroyo 1978) são auto-incompatíveis (sem detalhes sobre mecanismos de incompatibilidade). Em contraste, nas plantas do estrato herbáceo-arbustivo do cerrado, predominam sistemas xenogâmicos facultativos, com muitas espécies 
autocompatíveis (Barbosa 1997). É interessante, o fato de Casearia sylvestris, espécie arbustiva com estrutura floral semelhante a $C$. grandiflora e também polinizada por moscas, ser autocompatível (Barbosa 1997), corroborando este padrão geral.

Agradecimentos - À Cecília Lomônaco pela identificação e informações sobre a biologia das moscas. À Simone Carolina S. Silva pela preparação das pranchas e pela ajuda no trabalho de campo. À Ana Angélica Barbosa pelas sugestões durante a realização do trabalho e leitura crítica do manuscrito. $\mathrm{O}$ trabalho foi realizado no âmbito de um projeto integrado do CNPq (Processo 520872/96-7) que proveu uma bolsa de iniciação científica para a primeira autora.

\section{Referências bibliográficas}

ARRUDA, V.L.V. \& SAZIMA, M. 1996. Flores visitadas por sirfídeos (Diptera: Syrphidae) em mata mesófila de Campinas, SP. Revista Brasileira de Botânica 19:109-117.

BARBOSA, A.A.A.1997. Biologia reprodutiva de uma comunidade de campo sujo, Uberlândia/MG. Tese de doutorado, Universidade Estadual de Campinas, Campinas.

BARROSO, G.M., GUIMARÃES, E.F., ICHASO, C.L.F., COSTA, C.G., \& PEIXOTO, A.L. 1978. Sistemática de angiospermas do Brasil. Vol. I. Editora da Universidade de São Paulo, São Paulo.

BAWA, K.S. 1974. Breeding systems of tree species of a lowland tropical community. Evolution 28:85-92.

BAWA, K.S. 1990. Plant-pollinator interactions in tropical rain forest. Annual Review of Ecology and Systematics 21:399422.

CREPET, W.L. 1983. The role of insect pollination in the evolution of the angiosperms. In Pollination biology. (L. Real, ed). Academic Press, Orlando, p.29-50.

CRUDEN, R.W. 1977. Pollen-ovule ratios: a conservative indicator of breeding systems in flowering plants. Evolution 31:32-46.

ENDRESS, P.K. 1994. Diversity and evolutionary biology of tropical flowers. Cambridge University Press, Cambridge.

FAEGRI, K., \& Van der Pijl,L. 1979. The principles of pollination ecology. Pergamon Press, Oxford.

GIBBS, P.E., OLIVEIRA, P.E., \& BIANCHI, M. 1999. Post-zygotic control of selfing in Hymenaea stigonocarpa (Leguminosae - Caesalpinoideae), a bat-pollinated tree of the Brazilian cerrados. International Journal of Plant Science 160:72-78

GIBBS, P.E., \& BIANCHI, M.B. 1999. Does late-acting self-incompatibility (LSI) show family clustering? Two more species of Bignoniaceae with LSI: Dolichandra cynanchoides and Tabebuia nodosa. Annals of Botany 84:449-457.

GILBERT, F.S. 1981. Foraging ecology of hoverflies: morphology of the mouthparts in relation to feeding on nectar and pollen in some commom urban species. Ecological Entomology 6:245-262.
GILBERT, F.S. 1985. Diurnal activity patterns in hoverflies (Diptera, Syrphidae). Ecological Entomology 10:385-392.

GUILHERME, F.G., NAKAJIMA, J.N., PRADO-LIMA, C.A. \& VANINI, A. 1998. As fitofisionomias e a flora lenhosa nativas do Parque do Sabiá, Uberlândia-MG. Daphne $8(2): 17-30$.

INOUYE, D.W., FAVRE, N.D., LANUN, J.A., LEVINE, D.M., MEYERS, J.B., ROBERTS, M.S., TSAO, F.C., \& WANG, Y.Y. 1980. The effect of non-sugar nectar constituents on estimates of nectar energy content. Ecology 61:992-995.

KEARNS, C.A. \& INOUYE D.W. 1993. Techniques for pollination biologists. University Press of Colorado, Niwot.

KRESS, W.J., \& BEACH, J.H. 1994. Flowering plant reproductive systems. In La Selva: Ecology and Natural History of a Lowland Tropical Rainforest (L.A. McDade, K.S. Bawa, G.S. Hartshorn, \& H.A. Hespenheide, eds.). University of Chicago Press, Chicago. p.161-182.

MARTIN, F.N. 1959. Staining and observing pollen tubes in the style by means of fluorescence. Stain Technology 34:125.

NIC-LUGHADHA, E. 1998. Preferential outcrossing in Gomidesia (Myrtaceae) is maintained by a post-zygotic mechanism. In Reproductive Biology: in systematics, conservation and economic botany (S. Owens, \& P. Rudall, ed.). Royal Botanic Gardens, Kew, p.363-379.

OLIVEIRA, P.E. \& GIBBS, P.E. 2000. Reproductive biology of woody plants in a cerrado community of Central Brazil. Flora 195:(no prelo)

OLIVEIRA, P.E., GIBBS, P.E. \& BIANCHI. M. 1991. Pollination and breeding biology of Vellozia squamata (LilialesVelloziaceae) a species of the Brazilian cerrados. Botanica Acta 104:392-398.

OWEM, J. 1981. Throphic variety and abundance of hoverflies (Diptera, Syrphidae) in an english suburban garden. Holarctic Ecology 4:21-228.

POMBAL, E.C.P. 1994. Biologia floral de duas espécies dicogâmicas, Metrodorea nigra e M. stipularis (Rutaceae), em matas semidecídua no sudeste do Brasil. Dissertação de Mestrado, Universidade Estadual Paulista, Rio Claro, Rio Claro.

POMBAL, E.C.P. \& MORELLATO, P.C. 1995. Polinização por moscas em Dendropanax cuneatum Decne. \& Panch. (Araliaceae) em floresta semidecídua no sudeste do Brasil. Revista Brasileira de Botânica 18:157-162.

PROCTOR, M., YEO, P. \& LACK, A. 1996. The natural history of pollination. The Bath Press, London.

RADFORD, A.E., DICKISON, J.R. \& BELL, C.R. 1974. Vascular plant systematics. Harper \& Row Publishers, New York.

ROSA, R., LIMA, S.C. \& ASSUNÇÃO, W.L. 1991. Abordagem preliminar das condições climáticas de Uberlândia, MG. Sociedade \& Natureza 3:91-108.

RUIZ, T.Z., \& ARROYO, M.T.K. 1978. Plant reproductive ecology of a secondary deciduous tropical forest. Biotropica 10:221-230. 
SAGE, T.L., BERTIN, R.I., \& WILLIAMS, E.G. 1994. Ovarian and other late-acting self-incompatibility systems. In Genetic control of self-incompatibility and reproductive development in flowering plants (E.G. Williams, A.E. Clarke, \& R.B. Knox, eds.). Kluwer Academic Publishers, Netherlands. p.116-140.

SEAVEY, S.R. \& BAWA K.S. 1986. Late-acting self-incompatibility in Angiosperms. Botanical Review 52:195-219.

SILVA-JR., M.C., FELFILI, J.M., NOGUEIRA, P.E. \& REZENDE, A.V. 1998. Análise florística das matas de galeria do Distrito Federal. In Cerrado: Matas de Galeria (J.F. Ribeiro, ed.). EMBRAPA, Planaltina p.53-84.
TARODA, N., \& GIBBS, P.E. 1982. Floral biology and breeding system of Sterculia chicha S.Hil. (Sterculiaceae). New Phytologist 90:735-743.

VOCKEROTH, J.R. \& THOMPSON, F.C. 1987. Syrphidae. In Manual of Neartic Diptera. v.2. (J.F. McAlpine, ed.). (Monograph $n^{\circ} 28$ ). Research Branch Agriculture Canada, Ottawa.

VOGEL, S. 1983. Ecophysiology of zoophilic. In Physiological plant ecology III. Responses to the chemical and biological environment. (O.T. Lange, P.S. Nobel, C.B. Osmond \& H. Ziegler, eds). Springer-Verlag, Berlin, p.560-624.

WIENS, D., CALVIN, C.L., WILSON, C.A., DAVERN, C.I., FRANK, D., \& SEAVEY, S.R. 1987. Reproductive success, spontaneous embryo abortion and genetic load in flowering plants. Oecologia 71:501-509. 